Original Article

\title{
Impact of Severe Postoperative Complications after Cardiac Surgery on Mortality in Patients Aged over 80 Years
}

\author{
Hiroyuki Kamiya, MD, PhD, ${ }^{1}$ Nadine Tanzeem, MD,${ }^{2}$ Payam Akhyari, MD, ${ }^{1}$ \\ Anabel Pedraza, MD, ${ }^{2}$ Klaus Kallenbach, MD, PhD,${ }^{2}$ Artur Lichtenberg, MD, PhD, ${ }^{1}$ \\ and Matthias Karck, $\mathrm{MD}, \mathrm{PhD}^{2}$
}

\begin{abstract}
Background: The aims of this study are (1) to investigate the occurrence rate of postoperative complications in patients $\geq 80$ years old after cardiac surgery and (2) to elucidate the impact of the most common postoperative complications on mortality.

Methods: Between January 1998 and December 2007, 649 patients aged over 80 years received isolated first-time coronary artery bypass graft (CABG), isolated aortic valve replacement (AVR) or a combination of both in our institute. Prospectively entered patient data were analyzed with respect to major complications and outcome parameters.

Results: Acute renal failure (55.0\% vs. $7.5 \%, p=0.0001)$, low cardiac out-put syndrome (43.1\% vs. 8.8\%, $p=0.0001)$, sepsis $(52.0 \%$ vs. $10.3 \%, p=0.0001)$, prolonged respiratory failure with tracheotomy $(29.0 \%$ vs. $11.0 \%, p=0.002)$, re-thoracotomy due to bleeding (26.9\% vs. $10.6 \%, p=0.0001)$, and postoperative laparotomy $(30.8 \%$ vs. $11.5 \%$, $\mathbf{p}=\mathbf{0 . 0 3 3}$ ) had a significant impact on mortality. A multivariate analysis revealed that advanced age (OR 1.130, 95\% CI; 1.017-1.256, $p=0.023)$, low output syndrome (OR $5.094,95 \% \mathrm{CI}$; $1.1635-15.871, p=0.005)$, renal failure (OR 8.128, 95\% CI; 3.347-19.742, $\mathrm{p}=0.0001)$ and sepsis $(\mathrm{OR} 4.975,95 \% \mathrm{CI} ; 1.420-17.426, \mathrm{p}=\mathbf{0 . 0 1 2})$ as independent risk factors.

Conclusions: The present study demonstrates that among major complications, low output syndrome, renal failure requiring renal replacement therapy and sepsis, dramatically impaired the postoperative course patients aged over 80 years undergoing CABG, AVR or combined CABG and AVR.
\end{abstract}

Keywords: cardiac surgery, octogenarians, postoperative complication and mortality

\footnotetext{
${ }^{1}$ Department of Cardiovascular Surgery, Duesseldorf University Hospital, Duesseldorf, Germany

${ }^{2}$ Department of Cardiac Surgery, University Hospital Heidelberg, Heidelberg, Germany
}

Received: January 22, 2013; Accepted: May 31, 2013

Corresponding author: Hiroyuki Kamiya, MD, PhD. Department of Cardiovascular Surgery, Duesseldorf University Hospital, Moorenstrasse 5, Duesseldorf 40225, Germany Email: hiroyuki.kamiya@med.uni-duesseldorf.de (C)2014 The Editorial Committee of Annals of Thoracic and Cardiovascular Surgery. All rights reserved.

\section{Introduction}

Nowadays, cardiac surgery can be performed with an acceptable risk, even in octogenarians ${ }^{1-3)}$ and nonagenarians. However, studies have shown that older patients are, as a matter of course, more likely to suffer from postoperative complications, resulting in a higher mortality in this particular patient cohort. ${ }^{4-8)}$

It is widely accepted that the inferior outcome of elderly patients with major complications may be due to 
(1) an increased incidence of perioperative adverse events and (2) a limited overall systemic reserve to resist the biological challenge of major complications that may arise as a consequence of adverse events in the postoperative course. However, a detailed analysis of the individual impact of common postoperative adverse events and major complications, specifically in elderly patients, has not been performed so far.

The aims of this study are (1) to investigate the occurrence of postoperative complications in patients aged over 80 years after cardiac surgery and (2) to clarify the impact of individual postoperative complications on the mortality.

\section{Patients and Methods}

\section{Patients}

Between January 1998 and December 2007, 649 patients aged over 80 years received an isolated firsttime aortic valve replacement (AVR group, $n=179$ ), coronary artery bypass grafting (CABG group, $\mathrm{n}=311$ ), or CABG and AVR (Combi group, $n=159$ ) in our institute and were included in this study. Patient data on demographics, operative data, perioperative outcome data were prospectively entered in an institutional data registry. In addition, medical records of all patients were retrospectively reviewed for this study. This retrospective study was approved by the institutional ethical board.

\section{Definition of mortality and postoperative major complications}

In-hospital death and/or 30 days mortality was defined as 'mortality'. The need of intraoperative or postoperative intraaortic balloon pumping (IABP) was defined as 'postoperative low out-put syndrome'. The need of postoperative renal replacement therapy was defined as 'renal failure', irrespective of the applied system (e.g. continuous or intermittent hemofiltration or hemodiafiltration etc.). 'Prolonged respiratory failure' was defined as repetitive failure of respiratory weaning and necessity of prolonged ventilation beyond a week with the consecutive need for a tracheotomy. 'Stroke' was defined as any neurological deficit with either a positive finding on a cranial CT-scan or with prolonged neurological deficit beyond 24 hours. A need for re-thoracotomy due to bleeding was defined as 'postoperative bleeding'. Laparotomy was performed when an acute abdomen occurred. 'Sepsis' was defined as a combination of positive blood culture and/or imaging results positive for an infection (pneumonia in X-Ray etc.) and two of the following four criteria: (1) core body temperature $>38^{\circ} \mathrm{C}$ or $<36^{\circ} \mathrm{C}$ (2) heart rate $>90$ beats/min (3) respiratory rate $>20$ breaths/ min or $\mathrm{PaCO} 2<32$ torr $(<4.3 \mathrm{kPa})$ (4) white blood cells $>12.000$ cells $/ \mathrm{mm}^{3},<4.000$ cells $/ \mathrm{mm}^{3}$, or $>10 \%$ immature (band) forms. An infection in the region of the sternal wound associated with a sternal dehiscence and the need for a surgical intervention was defined as 'mediastinitis'.

\section{Statistical analysis}

Results are expressed as mean \pm standard deviation. Statistical analysis was performed using Student's t-test or one-way ANOVA for continuous variables or $\chi^{2}$ tests (Fisher's exact tests if $\mathrm{n}<5$ ) for categorical variables. Logistic regression was also used for the multivariate analysis of risk factors for mortality. A p value less than 0.05 was considered significant. All statistical analyses were performed using SPSS 16.0 software (SPSS Inc., Chicago, Illinois, USA).

\section{Results}

Patient characteristics and data on the postoperative course are presented in Tables 1 and 2, respectively. Not surprisingly, there were significant differences in patient characteristics and intraoperative parameters among the 3 study groups. However, there was no difference among groups concerning the rate of severe complications except for postoperative low-output syndrome requiring an intraaortic balloon pumping, occurring most frequently in AVR group. To focus on the main purpose of this study, all patients were together analyzed further.

\section{Impact of complications on mortality}

The impact of each major complication on the mortality is demonstrated in Fig. 1. Acute renal failure requiring a renal replacement therapy $(55.0 \%$ vs. $7.5 \%, \mathrm{p}=$ $0.0001)$, low out-put syndrome requiring an IABP-support $(43.1 \%$ vs. $8.8 \%, \mathrm{p}=0.0001)$, sepsis $(52.0 \%$ vs. $10.3 \%, \mathrm{p}=0.0001)$, prolonged respiratory failure with tracheotomy had $(29.0 \%$ vs. $11.0 \%, \mathrm{p}=0.002)$, Re-thoracotomy due to bleeding $(26.9 \%$ vs. $10.6 \%, \mathrm{p}=$ $0.0001)$, postoperative laparotomy $(30.8 \%$ vs. $11.5 \%$, $\mathrm{p}=0.033)$ had a significant impact on mortality, but stroke $(0 \%$ vs. $12 \%, \mathrm{p}=0.329)$ and mediastinitis $(10.5 \%$ vs. $11.9 \%, p=0.885)$ were no predicting factor for mortality. 
Table 1 Patient characteristics in each group

\begin{tabular}{|c|c|c|c|c|c|}
\hline Preoperative parameters & Total $(n=649)$ & $\begin{array}{l}\text { AVR group } \\
(\mathrm{n}=179)\end{array}$ & $\begin{array}{l}\text { CABG group } \\
\quad(\mathrm{n}=311)\end{array}$ & $\begin{array}{l}\text { Combi group } \\
\quad(\mathrm{n}=159)\end{array}$ & $\mathrm{p}$ \\
\hline Age (years) & $82.5 \pm 3.0$ & $81.9 \pm 2.0$ & $81.9 \pm 2.0$ & $82.8 \pm 2.8$ & $\begin{array}{l}\text { AVR vs CABG } p=0.0001 \\
\text { CABG vs Combi } p=0.0001 \\
\text { AVR vs Combi } P=0.336\end{array}$ \\
\hline Male (n) & $319(49.2 \%)$ & $55(30.7 \%)$ & $188(60.5 \%)$ & $76(47.8 \%)$ & $\begin{array}{l}\text { AVR vs CABG } p=0.0001 \\
\text { CABG vs Combi } p=0.009 \\
\text { AVR vs Combi } p=0.001\end{array}$ \\
\hline $\operatorname{BMI}\left(\mathrm{kg} / \mathrm{m}^{2}\right)$ & $25.8 \pm 3.7$ & $25.4 \pm 3.7$ & $25.9 \pm 3.6$ & $25.9 \pm 3.6$ & $\begin{array}{l}\text { AVR vs CABG } p=0.122 \\
\text { CABG vs Combi } p=0.808 \\
\text { AVR vs Combi } p=0.264\end{array}$ \\
\hline Diabetes mellitus (n) & $218(33.6 \%)$ & $51(28.5 \%)$ & $118(37.9 \%)$ & $49(30.8 \%)$ & $\begin{array}{l}\text { AVR vs CABG } p=0.034 \\
\text { CABG vs Combi } p=0.127 \\
\text { AVR vs Combi } p=0.640\end{array}$ \\
\hline Hypertention (n) & $582(89.7 \%)$ & $156(87.2 \%)$ & $286(91.7 \%)$ & $140(88 \%)$ & $\begin{array}{l}\text { AVR vs CABG } p=0.085 \\
\text { CABG vs Combi } p=0.168 \\
\text { AVR vs Combi } p=0.802\end{array}$ \\
\hline Hyperlipidemia (n) & $435(67.0 \%)$ & $90(50.3 \%)$ & $238(76.5 \%)$ & $107(67.3 \%)$ & $\begin{array}{l}\text { AVR vs CABG } p=0.0001 \\
\text { CABG vs Combi } p=0.032 \\
\text { AVR vs Combi } p=0.002\end{array}$ \\
\hline Current smoker (n) & $39(6.0 \%)$ & $6(3.4 \%)$ & $27(8.7 \%)$ & $6(3.8 \%)$ & $\begin{array}{l}\text { AVR vs CABG } p=0.056 \\
\text { CABG vs Combi } p=0.130 \\
\text { AVR vs Combi } p=0.555\end{array}$ \\
\hline Pulmonary hypertension (n) & $119(18.3 \%)$ & $47(26.3 \%)$ & $19(6.1 \%)$ & $53(33.3 \%)$ & $\begin{array}{l}\text { AVR vs CABG } p=0.0001 \\
\text { CABG vs Combi } p=0.0001 \\
\text { AVR vs Combi } p=0.155\end{array}$ \\
\hline NYHA class IV (n) & $231(35.6 \%)$ & $50(27.9 \%)$ & $123(39.5 \%)$ & $58(36.5 \%)$ & $\begin{array}{l}\text { AVR vs CABG } p=0.007 \\
\text { CABG vs Combi } p=0.610 \\
\text { AVR vs Combi } p=0.056\end{array}$ \\
\hline $\mathrm{EF}(\%)$ & $59.3 \pm 16.3$ & $60.1 \pm 15.7$ & $60.2 \pm 15.6$ & $57.0 \pm 18.1$ & $\begin{array}{l}\text { AVR vs CABG } p=0.989 \\
\text { CABG vs Combi } p=0.325 \\
\text { AVR vs Combi } p=0.385\end{array}$ \\
\hline $\mathrm{EF}<40 \%(\mathrm{n})$ & $192(29.6 \%)$ & $40(23.1 \%)$ & $105(36.3 \%)$ & $47(32.6 \%)$ & $\begin{array}{l}\text { AVR vs CABG } p=0.003 \\
\text { CABG vs Combi } p=0.284 \\
\text { AVR vs Combi } p=0.085\end{array}$ \\
\hline Previous infarction (n) & $248(38.2 \%)$ & $22(12.3 \%)$ & $175(56.3 \%)$ & $51(32 \%)$ & $\begin{array}{l}\text { AVR vs CABG } p=0.0001 \\
\text { CABG vs Combi } p=0.0001 \\
\text { AVR vs Combi } p=0.0001\end{array}$ \\
\hline Creatine kinase (U/L) & $88.9 \pm 150$ & $68.4 \pm 52.2$ & $110.2 \pm 208.2$ & $73.2 \pm 71.4$ & $\begin{array}{l}\text { AVR vs CABG } p=0.010 \\
\text { CABG vs Combi } p=0.033 \\
\text { AVR vs Combi } p=0.799\end{array}$ \\
\hline Preoperative IABP & $32(4.9 \%)$ & $1(0.6 \%)$ & $25(8.0 \%)$ & $6(3.7 \%)$ & $\begin{array}{l}\text { AVR vs CABG } p=0.0001 \\
\text { CABG vs Combi } p=0.078 \\
\text { AVR vs Combi } p=0.038\end{array}$ \\
\hline Emergency (n) & $62(9.6 \%)$ & $9(5.0 \%)$ & $44(14.2 \%)$ & $9(5.7 \%)$ & $\begin{array}{l}\text { AVR vs CABG } p=0.001 \\
\text { CABG vs Combi } p=0.023 \\
\text { AVR vs Combi } p=0.164\end{array}$ \\
\hline Preoperative ventilation (n) & $6(0.9 \%)$ & $2(1.1 \%)$ & $3(1 \%)$ & $1(0.6 \%)$ & $\begin{array}{l}\text { AVR vs CABG } p=0.911 \\
\text { CABG vs Combi } p=0.683 \\
\text { AVR vs Combi } p=0.683\end{array}$ \\
\hline Preoperative Creatinine (mg/dl) & $1.1 \pm 0.6$ & $1.0 \pm 0.6$ & $1.2 \pm 0.5$ & $1.2 \pm 0.9$ & $\begin{array}{l}\text { AVR vs CABG } p=0.025 \\
\text { CABG vs Combi } p=0.530 \\
\text { AVR vs Combi } p=0.013\end{array}$ \\
\hline Preoperative dialysis (n) & $5(0.8 \%)$ & $2(1.1 \%)$ & $2(0.6 \%)$ & $1(0.6 \%)$ & $\begin{array}{l}\text { AVR vs CABG } p=0.574 \\
\text { CABG vs Combi } p=0.985 \\
\text { AVR vs Combi } p=0.633\end{array}$ \\
\hline
\end{tabular}

(Continued) 
Table 1 (Continued)

\begin{tabular}{|c|c|c|c|c|c|}
\hline Preoperative parameters & Total $(n=649)$ & $\begin{array}{l}\text { AVR group } \\
(\mathrm{n}=179)\end{array}$ & $\begin{array}{l}\text { CABG group } \\
\quad(n=311)\end{array}$ & $\begin{array}{l}\text { Combi group } \\
\quad(\mathrm{n}=159)\end{array}$ & $\mathrm{p}$ \\
\hline Peripheral arterial disease (n) & $133(22.5 \%)$ & $32(17.9 \%)$ & $67(21.5 \%)$ & $34(21.4 \%)$ & $\begin{array}{l}\text { AVR vs CABG } p=0.330 \\
\text { CABG vs Combi } p=0.968 \\
\text { AVR vs Combi } p=0.417\end{array}$ \\
\hline COPD (n) & $145(22.5 \%)$ & $41(22.9 \%)$ & $64(20.6 \%)$ & $40(25.2 \%)$ & $\begin{array}{l}\text { AVR vs CABG } p=0.558 \\
\text { CABG vs Combi } p=0.266 \\
\text { AVR vs Combi } p=0.628\end{array}$ \\
\hline \multicolumn{6}{|l|}{ Intraoperative parameters } \\
\hline Operation time (min) & $199.1 \pm 66.1$ & $175.9 \pm 51.2$ & $197.5 \pm 66.5$ & $228.3 \pm 69.5$ & $\begin{array}{l}\text { AVR vs CABG } p=0.0001 \\
\text { CABG vs Combi } p=0.0001 \\
\text { AVR vs Combi } p=0.0001\end{array}$ \\
\hline CPB time (min) & $101 \pm 38.6$ & $93.1 \pm 28$ & $92.8 \pm 37.5$ & $125.8 \pm 40.5$ & $\begin{array}{l}\text { AVR vs CABG } p=0.928 \\
\text { CABG vs Combi } p=0.0001 \\
\text { AVR vs Combi } p=0.0001\end{array}$ \\
\hline $\mathrm{X}$-clamp time (min) & $58.7 \pm 21.2$ & $59 \pm 13.1$ & $48.4 \pm 18.9$ & $78.2 \pm 19$ & $\begin{array}{l}\text { AVR vs CABG } p=0.0001 \\
\text { CABG vs Combi } p=0.0001 \\
\text { AVR vs Combi } p=0.0001\end{array}$ \\
\hline Mean size of prosthesis (mm) & & $21.8 \pm 1.6$ & & $22.3 \pm 1.6$ & AVR vs Combi $p=0.005$ \\
\hline Number of grafts & & & $2.9 \pm 0.9$ & $1.7 \pm 0.9$ & CABG vs Combi $p=0.0001$ \\
\hline
\end{tabular}

Table 2 Occurrence of severe complications

\begin{tabular}{|c|c|c|c|c|c|}
\hline & $\begin{array}{c}\text { Total } \\
(\mathrm{n}=649)\end{array}$ & $\begin{array}{l}\text { AVR group } \\
(\mathrm{n}=179)\end{array}$ & $\begin{array}{l}\text { CABG group } \\
\quad(\mathrm{n}=311)\end{array}$ & $\begin{array}{l}\text { Combi group } \\
\quad(\mathrm{n}=159)\end{array}$ & $\mathrm{p}$ \\
\hline $\begin{array}{l}\text { Low output syndrome } \\
\text { requiring IABP-support (n) }\end{array}$ & $35(3.5 \%)$ & $2(1.1 \%)$ & $17(5.5 \%)$ & $4(2.5 \%)$ & $\begin{array}{l}\text { AVR vs CABG } p=0.016 \\
\text { CABG vs Combi } p=0.143 \\
\text { AVR vs Combi } p=0.331\end{array}$ \\
\hline Postoperative dialysis (n) & $60(9.2 \%)$ & $15(8.4 \%)$ & $27(8.7 \%)$ & $18(11.3 \%)$ & $\begin{array}{l}\text { AVR vs CABG } p=0.909 \\
\text { CABG vs Combi } p=0.358 \\
\text { AVR vs Combi } p=0.363\end{array}$ \\
\hline Tracheotomy (n) & $31(4.8 \%)$ & $7(3.9 \%)$ & $15(4.8 \%)$ & $9(5.7 \%)$ & $\begin{array}{l}\text { AVR vs CABG } p=0.639 \\
\text { CABG vs Combi } p=0.358 \\
\text { AVR vs Combi } p=0.450\end{array}$ \\
\hline Stroke (n) & $7(1.1 \%)$ & $3(1.7 \%)$ & $4(1.3 \%)$ & $0(0 \%)$ & $\begin{array}{l}\text { AVR vs CABG } p=0.726 \\
\text { CABG vs Combi } p=0.151 \\
\text { AVR vs Combi } p=0.101\end{array}$ \\
\hline Mediastinitis (n) & $19(2.9 \%)$ & $4(2.2 \%)$ & $9(2.9 \%)$ & $6(3.8 \%)$ & $\begin{array}{l}\text { AVR vs CABG } p=0.662 \\
\text { CABG vs Combi } p=0.608 \\
\text { AVR vs Combi } p=0.404\end{array}$ \\
\hline $\begin{array}{l}\text { Re-thoracotomy due to } \\
\text { bleeding (n) }\end{array}$ & $52(8.0 \%)$ & $13(7.3 \%)$ & $24(7.7 \%)$ & $15(9.4 \%)$ & $\begin{array}{l}\text { AVR vs CABG } p=0.855 \\
\text { CABG vs Combi } p=0.523 \\
\text { AVR vs Combi } p=0.470\end{array}$ \\
\hline Laparotomy (n) & $13(2.0 \%)$ & $4(2.2 \%)$ & $7(2.3 \%)$ & $2(1.3 \%)$ & $\begin{array}{l}\text { AVR vs CABG } p=0.991 \\
\text { CABG vs Combi } p=0.457 \\
\text { AVR vs Combi } p=0.497\end{array}$ \\
\hline Sepsis (n) & $25(3.9 \%)$ & $8(4.5 \%)$ & $12(3.9 \%)$ & $5(3.1 \%)$ & $\begin{array}{l}\text { AVR vs CABG } p=0.742 \\
\text { CABG vs Combi } p=0.695 \\
\text { AVR vs Combi } p=0.527\end{array}$ \\
\hline $\begin{array}{l}\text { Duration of ventilation } \\
\text { (hours) }\end{array}$ & $61.8 \pm 168.1$ & $47.0 \pm 113.3$ & $65.3 \pm 195.3$ & $61.8 \pm 168$ & $\begin{array}{l}\text { AVR vs CABG } p=0.251 \\
\text { CABG vs Combi } p=0.700 \\
\text { AVR vs Combi } p=0.182\end{array}$ \\
\hline ICU stay (days) & $5.0 \pm 8.0$ & $4.6 \pm 7.2$ & $5.2 \pm 9.2$ & $5.4 \pm 7.5$ & $\begin{array}{l}\text { AVR vs CABG } p=0.423 \\
\text { CABG vs Combi } p=0.845 \\
\text { AVR vs Combi } p=0.387\end{array}$ \\
\hline Mortality & $77(11.9 \%)$ & $15(8.4 \%)$ & $43(13.8 \%)$ & $19(11.9 \%)$ & $\begin{array}{l}\text { AVR vs CABG } p=0.072 \\
\text { CABG vs Combi } p=0.569 \\
\text { AVR vs Combi } p=0.276\end{array}$ \\
\hline
\end{tabular}




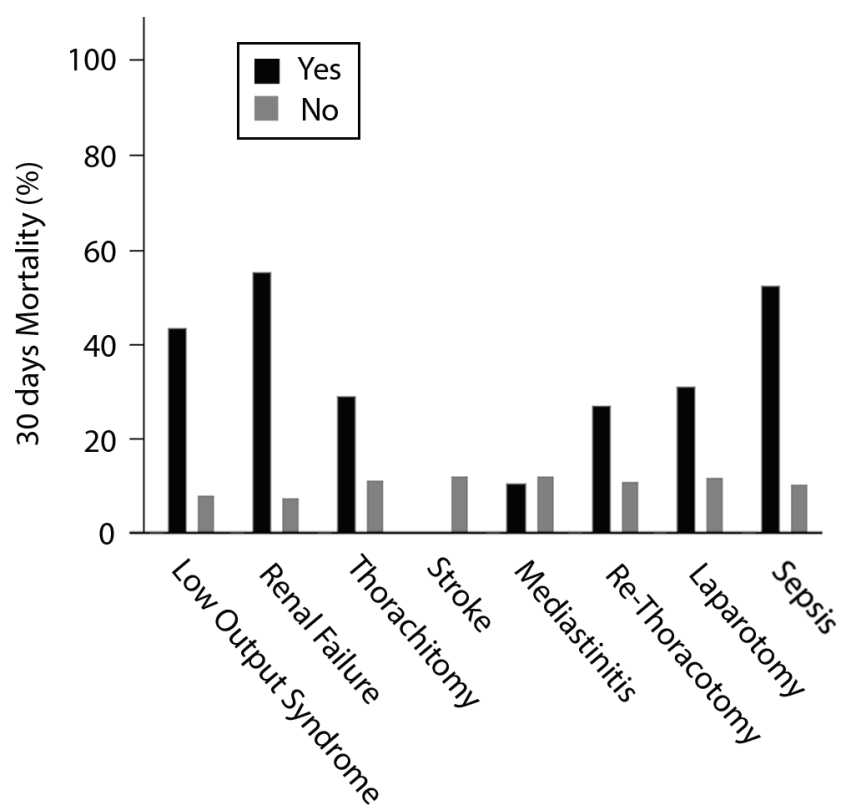

Fig. 1 Mortality in patients with or without severe complications.

\section{Univariate analysis for early mortality}

Univariate analysis revealed the following parameters as significant risk factors (Table 3): age $(p=0.002)$, ejection fraction $\leq 40 \%(\mathrm{p}=0.003)$, use of preoperative $\operatorname{IABP}(p=0.0001)$, preoperative ventilation $(\mathrm{p}=0.0001)$, preoperative renal impairment $(\mathrm{p}=0.0001)$, duration of the operation $(p=0.0001)$, cardiopulmonary bypass time $(\mathrm{p}=0.0001)$, aortic cross-clamp time $(\mathrm{p}=0.01)$, and postoperative need of epinephrine $(\mathrm{p}=0.0001)$, postoperative low output syndrome $(\mathrm{p}=0.0001)$, postoperative renal failure $(\mathrm{p}=0.0001)$, tracheotomy $(\mathrm{p}=0.002)$, re-thoracotomy due to bleeding $(\mathrm{p}=0.0001)$, Laparotomy $(p=0.033)$ and sepsis $(p=0.0001)$. Surprisingly, there were more patients with arterial hypertension among the survivors as compared to patients in the 30-d-mortality group $(\mathrm{p}=0.016)$.

\section{Multivariate analysis of preoperative factors for early mortality and severe complications}

As composite events including death and severe complications (low output syndrome requiring IABP-support, postoperative dialysis, thorachiotomy, stroke, mediastinitis, re-thoracotomy due to bleeding, laparotomy and sepsis), 193 patients had any event. Preoperative factors were multivariately analysed for those composite events. Age (OR 1.22, 95\%CI; 1.015-1.381, p = 0.01), EF <0\% (OR 1.08, 95\% CI; 1.005-1.384, $\mathrm{p}=0.04)$ and preoperative creatinine (OR 1.23, 95\%CI; 1.121-3.578, $\mathrm{p}=0.01$ ) were independent risk factors.
Multivariate analysis of all factors for early mortality

All factors including preoperative, intraoperative and postoperative factors were included into multivariate analysis and the results are listed in Table 4. Age was revealed as the only preoperative independent risk factor beside the other risk factors represented by the major postoperative complications.

\section{Discussion}

The crucial findings of the present study were: (1) simple cardiac surgery such as AVR, CABG or AVR + CABG could be performed with acceptable results even in very elderly patients, especially when no postoperative complications occurred, (2) among major postoperative complications, low out-put syndrome, renal failure requiring renal replacement therapy and sepsis impaired significantly postoperative outcome and these three postoperative complications were independent risk factors and (3) other pre- and intraoperative parameters were no independent risk factors except for an advanced age at the time of operation.

Advances in surgical procedures, anaesthetic techniques and intensive care therapy have pushed the limit of age dramatically over the past decade. Many previous studies have demonstrated that cardiac surgery can be performed with acceptable results not only in octogenarians, but also in nonagenarians. ${ }^{9-11)}$

However, it is well known that advanced age is a risk factor for mortality, and elderly patients are more likely to suffer from postoperative complications. ${ }^{2,3,8,9)}$ Bridges, et al. reported from the Society of Thoracic Surgeons National Database with a total of 662033 patients that operative mortality was $11.8 \%$ for patients more than 90 years of age, $7.1 \%$ for those 80 to 89 years, and $2.8 \%$ for those 50 to 79 years and the incidence of renal failure and prolonged ventilation was highest among patients more than 90 years of age (9.2\% and $12.2 \%)$, compared with those 80 to 89 years $(7.7 \%$ and $10.5 \%)$ or 50 to 79 years $(3.5 \%$ and $\left.6.0 \%) .{ }^{9}\right)$ In contrast to their study, mortality in the present study was high as $12 \%$. It may be due to delayed operation in our patient cohort as the prevalence of NYHA IV patients to be $36 \%$.

Previous reports have focused on the prediction of overall mortality and morbidity, but the impact of each complication on mortality has not been well studied. This issue is particularly important in the elderly patients because, as the elderly patients tend to have less tolerance to postoperative complications. 
Table 3 Univariate analysis for $\mathbf{3 0}$ days mortality

\begin{tabular}{|c|c|c|c|}
\hline & $\begin{array}{l}\text { Dead within } 30 \text { days } \\
\qquad(\mathrm{n}=77)\end{array}$ & $\begin{array}{l}\text { Survivor } \\
(\mathrm{n}=572)\end{array}$ & $\mathrm{p}$ \\
\hline Age (years) & $83.1 \pm 3.3$ & $82.4 \pm 2.4$ & 0.002 \\
\hline Male (n) & $39(50.6 \%)$ & $280(48.9 \%)$ & 0.780 \\
\hline $\mathrm{BMI}\left(\mathrm{kg} / \mathrm{m}^{2}\right)$ & $25.4 \pm 3.8$ & $25.8 \pm 3.6$ & 0.313 \\
\hline Diabetes mellitus (n) & $30(39.0 \%)$ & $188(32.9 \%)$ & 0.288 \\
\hline Hypertention (n) & $63(81.8 \%)$ & $519(90.7 \%)$ & 0.016 \\
\hline Hyperlipidemia (n) & $47(61 \%)$ & $388(67.8 \%)$ & 0.234 \\
\hline Current smoker (n) & $7(9 \%)$ & $32(5.6 \%)$ & 0.423 \\
\hline Pulmonary hypertension (n) & $12(12.6 \%)$ & $107(18.7 \%)$ & 0.506 \\
\hline NYHA class IV (n) & $33(42.9 \%)$ & $198(34.6 \%)$ & 0.113 \\
\hline $\mathrm{EF}<40 \%(\mathrm{n})$ & $14(20.3 \%)$ & $48(8.9 \%)$ & 0.003 \\
\hline Previous infarction (n) & $33(42.9 \%)$ & $215(36.6 \%)$ & 0.234 \\
\hline Creatine kinase (U/L) & $111 \pm 209.4$ & $87 \pm 143.2$ & 0.331 \\
\hline Preoprative IABP (n) & $11(14.3 \%)$ & $21(3.7 \%)$ & 0.0001 \\
\hline Emergency (n) & $9(11.7 \%)$ & $53(9.3 \%)$ & 0.498 \\
\hline Preoperative ventilation (n) & $4(5.6 \%)$ & $2(0.4 \%)$ & 0.0001 \\
\hline Preoperative Creatinine (mg/dl) & $1.4 \pm 1.2$ & $1.1 \pm 0.5$ & 0.0001 \\
\hline Preoperative dialysis (n) & $1(1.3 \%)$ & $4(0.7 \%)$ & 0.572 \\
\hline Peripheral arterial disease (n) & $16(20.8 \%)$ & $117(20.5 \%)$ & 0.947 \\
\hline COPD (n) & $17(22 \%)$ & $128(22.4 \%)$ & 0.947 \\
\hline Operation time (min) & $247.5 \pm 91.8$ & $192.6 \pm 59$ & 0.0001 \\
\hline CPB time $(\min )$ & $124.7 \pm 56.8$ & $97.8 \pm 34.2$ & 0.0001 \\
\hline $\mathrm{X}$-clamp time $(\mathrm{min})$ & $62.9 \pm 24.9$ & $58.1 \pm 20.6$ & 0.01 \\
\hline Need of Epinephrine & $14(18.2 \%)$ & $24(4.2 \%)$ & 0.0001 \\
\hline Low output syndrome requiring & $25(32.5 \%)$ & $33(6.8 \%)$ & 0.0001 \\
\hline \multicolumn{4}{|l|}{ IABP-support (n) } \\
\hline Postoperative dialysis (n) & $33(42.9 \%)$ & $27(4.7 \%)$ & 0.0001 \\
\hline Tracheotomy (n) & $9(11.7 \%)$ & $22(3.9 \%)$ & 0.002 \\
\hline Stroke $(\mathrm{n})$ & $0(0 \%)$ & $7(1.2 \%)$ & 0.329 \\
\hline Mediastinitis (n) & $2(2.6 \%)$ & $17(3 \%)$ & 0.855 \\
\hline Re-thoracotomy due to bleeding (n) & $14(18.2 \%)$ & $38(6.6 \%)$ & 0.0001 \\
\hline Laparotomy (n) & $4(5.2 \%)$ & $9(1.6 \%)$ & 0.033 \\
\hline Sepsis & $13(16.9 \%)$ & $12(2.1 \%)$ & 0.0001 \\
\hline
\end{tabular}

Table 4 Multivariate analysis

\begin{tabular}{lccl}
\hline & OR & \multicolumn{1}{c}{$95 \%$ CI } & \multicolumn{1}{c}{$\mathrm{p}$} \\
\hline Age & 1.130 & $1.017-1.256$ & 0.023 \\
Low output syndrome & 5.094 & $1.635-15.871$ & 0.005 \\
Renal failure & 8.128 & $3.347-19.742$ & 0.0001 \\
Sepsis & 4.975 & $1.420-17.426$ & 0.012 \\
\hline
\end{tabular}

In the present study, postoperative renal failure requiring renal replacement therapy (55\% mortality), postoperative sepsis (52\% mortality), low output syndrome requiring intraaortic balloon pumping (43\% mortality), postoperative laparotomy due to abdominal complications (31\% mortality), prolonged respiratory insufficiency needing tracheotomy (29\% mortality) and re-thoracotomy due to postoperative bleeding ( $27 \%$ mortality) were significant risk factors. Among these risk factors only renal failure, sepsis and low output syndrome prove as independent risk factors in our cohort. This finding suggests that in the course of preoperative patient selection and patient preparation a higher level of awareness with respect to these complications is warranted, as they represent life-threatening postoperative events in very elderly patient cohort and any effort should be done to avoid those complications.

\section{Conclusion}

The present study demonstrates that among major complications, low output syndrome, renal failure 
requiring renal replacement therapy and sepsis, each impaired dramatically the postoperative course in patients undergoing cardiac surgery at an age of over 80 years.

\section{Disclosure Statement}

All authors have no conflict of interest.

\section{References}

1) Sheridan BC, Stearns SC, Rossi JS, et al. Three-year outcomes of multivessel revascularization in very elderly acute coronary syndrome patients. Ann Thorac Surg 2010; 89: 1889-94; discussion 1894-5.

2) Chaturvedi RK, Blaise M, Verdon J, et al. Cardiac surgery in octogenarians: long-term survival, functional status, living arrangements, and leisure activities. Ann Thorac Surg 2010; 89: 805-10.

3) Nissinen J, Wistbacka JO, Loponen P, et al. Coronary artery bypass surgery in octogenarians: long-term outcome can be better than expected. Ann Thorac Surg 2010; 89: 1119-24.
4) Avery GJ, Ley SJ, Hill JD, et al. Cardiac surgery in the octogenarian: evaluation of risk, cost, and outcome. Ann Thorac Surg 2001; 71: 591-6.

5) Rady MY, Ryan T, Starr NJ. Perioperative determinants of morbidity and mortality in elderly patients undergoing cardiac surgery. Crit Care Med 1998; 26: 225-35.

6) Piccione W. Cardiac surgery in the elderly: what have we learned? Crit Care Med 1998; 26: 196-7.

7) Schurr P, Boeken U, Litmathe J, et al. Predictors of postoperative complications in octogenarians undergoing cardiac surgery. Thorac Cardiovasc Surg 2010; 58: $200-3$.

8) Chee JH, Filion KB, Haider S, et al. Impact of age on hospital course and cost of coronary artery bypass grafting. Am J Cardiol 2004; 93: 768-71.

9) Bridges CR, Edwards FH, Peterson ED, et al. Cardiac surgery in nonagenarians and centenarians. J Am Coll Surg 2003; 197: 347-56; discussion 356-7.

10) Speziale G, Nasso G, Barattoni MC, et al. Operative and middle-term results of cardiac surgery in nonagenarians: a bridge toward routine practice. Circulation 2010; 121: 208-13.

11) Samuels LE, Sharma S, Morris RJ, et al. Cardiac surgery in nonagenarians. J Card Surg 1996; 11: 121-7. 